\section{LED Light Source: Major Advance in Fluorescence Microscopy}

\author{
Becky Hohman \\ Carl Zeiss MicroImaging, Inc. \\ Thornwood, NY \\ bhohman@zeiss.com
}

\section{A Radically New Approach}

Fluorescence microscopy requires an intense light source at the specific wavelength that will excite fluorescent dyes and proteins. The traditional method employs a white light, typically from a Mercury or Xenon arc lamp. Although such broad-spectrum lamps can generate ample light at desired wavelengths, only a small percentage of the projected light is useful in any particular application. The other wavelengths need to be suppressed to avoid background noise that reduces image contrast and obscures the fluorescent light emissions.

This process of suppressing extraneous light is complex, expensive, and only partially effective: even after decades of refinements, the best filters are not $100 \%$ percent successful at blocking the bleed through of non-specific photons. Some mitigation techniques end up not only suppressing peripheral light, but also significantly diminishing the intensity of the desired wavelengths. To address the root cause of the problem - the presence of non-specific photons - a radically different approach is coming to light.

Recent advances in high performance Light Emitting Diode (LED) technology have enabled the practical implementation of this theoretical model. High-intensity monochromatic LEDs are now available in a variety of colors that match the excitation bandwidth of many commonly used fluorescent dyes and proteins.

Carl Zeiss MicroImaging has incorporated this new LED technology in the Colibri illumination system, a light source system for widefield fluorescence microscopy that uses specific wavelength windows with much less need to suppress unwanted peripheral wavelengths from a white light arc lamp. The modular Colibri system employs up to four LEDs, each individually and instantly controlled by electrical current without any of the mechanical switching devices like filter wheels or shutters required by traditional illumination systems. LEDs of different colors can be used in combination, giving users the option of seeing multiple fluorochromes simultaneously or rapidly capturing sequential images of each fluorochrome.

\section{Light Source Requirements}

The most basic requirement of a fluorescence microscopy light source is closely matching the excitation wavelength of the fluorochrome to achieve a high contrast image-that is, an image with a high signal-to-noise ratio. Wavelengths that match the fluorochrome strengthen the signal, but any peripheral wavelengths produce background noise that can overshadow the signal emitted by the object of interest.

A second and related requirement is illumination intensity, i.e., the number of photons specific to the excitation wavelength that reach the specimen. The human eye is less sensitive than most automated detection systems, and therefore applications involving visual observation typically require higher levels of illumination intensity. On the other hand, lower intensity is necessary for live cell imaging applications to protect against photobleaching and phototoxicity.

Intensity of illumination and signal intensity do not just follow a linear correlation. Saturation effects and dark states become more and more important with increasing illumination intensity.
An additional consideration is protection of the spe wim copecially in live cell imaging applications. We have already mentioned the dangers of overexposure to light: phototoxicity of the specimen, and photobleaching of the fluorescent dye or protein. Overexposure can be avoided by attenuating the light intensity and by limiting the duration of the illumination to exactly the exposure time of the sensor. The cells also need to be protected from the heat generated by light source lamps and from the vibrations caused by mechanical filtering and switching devices.

Another factor to consider when evaluating light sources is the lifetime and stability of the lamp. Some light sources exhibit short-term intensity fluctuations and a substantial deterioration of performance over time. Limited lifespan results in more frequent bulb replacements, and poor stability diminishes the reproducibility of illumination conditions.

\section{Light-Emitting Diodes}

A light-emitting diode is a compact semiconductor device that emits incoherent narrow-spectrum light when electrical current is applied. The color of the emitted light depends on the composition and condition of the semiconducting material used, and can be nearultraviolet, visible, or infrared.

Unlike arc lamps that are inherently very bright, LED technology has dramatically evolved from humble beginnings. When the first commercial LEDs were introduced in 1968, they were capable of providing only 0.001 lumens of red light, a level of brightness suitable only for use as indicators. During the past four decades, LED technology has advanced at a rapid pace, comparable to the rate of advancement of microprocessors.

High performance LEDs are employed in many industrial, medical and military applications; for example, the Fiber Optic Gyroscope (FOG) is used in navigation, robotics and machine vision systems for automated manufacturing.

\section{Advantages of LED in Fluorescence Microscopy}

Now that high-performance LEDs provide sufficient intensity at the specific wavelengths required for many applications, fluorescence microscopy is able to take advantage of the benefits of LEDs, including their compact size, low power consumption, minimal heat output, fast switching and adjusting properties, high emission stability and extremely long life span.

The Colibri LED light source system from Carl Zeiss MicroImaging employs a flexible modular design and up to four LED modules that can be used simultaneously in Colibri. The beam paths from each module are steered into the microscope with a series of beam combiners, and 11 different LED modules are currently available for Colibri, from UV to dark red and including a broad-band Neutral White Light LED.

$\begin{array}{ccc}11 & \text { different LED modules from UV to dark red available } \\ 365 \mathrm{~nm} & 470 \mathrm{~nm} & 590 \mathrm{~nm} \\ 380 \mathrm{~nm} & 505 \mathrm{~nm} & 615 \mathrm{~nm} \\ 400 \mathrm{~nm} & 525 \text { to } 650 \mathrm{~nm} & 625 \mathrm{~nm} \\ 455 \mathrm{~nm} & 530 \mathrm{~nm} & \end{array}$

The modules can be easily exchanged by a user depending upon the experimental design of the day. The intensity of each module can be adjusted independently, precisely and reproducibly in percentage steps, so that for every fluorescent dye, the output emitted is precisely 
that which is needed to achieve the best possible compromise between the required excitation intensity and maximum sample protection. The modular design makes it possible to easily implement and exploit further developments in LED technology in the future.

An advantage of LEDs is that they instantly illuminate at full intensity as soon as electrical current is applied. Unlike arc lamps that are turned on continuously, LEDs can be switched on or off instantly when needed with no deleterious effects to their lifespan. Additionally, with no moving parts, the all-electronic system is vibration free.

Colibri is particularly well suited for imaging applications requiring fast switching between wavelengths. Only some 300 microseconds are needed to switch between the LED modules.

The intensity of every LED module can be adjusted in percentage steps, enabling equidistant multichannel images to be easily realized in time-lapse-series. Instead of adapting the integration times of the camera to the illumination intensity, LED technology makes it possible to set the illumination intensity for the required integration time. The LED illumination intensity is also highly stable over time, making quantitative analyses easier and more reliable.

The performance of an imaging system depends not only on the performance of each individual component, such as the light source, but on the sum of all factors making up such a system. The system software is a critical, but often neglected, component. In order to take full advantage of the Colibri LED light source technology, Carl Zeiss has integrated Colibri with its AxioVision imaging platform.

For live cell imaging, LED technology is ideally suited to the fast acquisition framework of AxioVision which forms the backbone of the Cell Observer HS system (released in 2005). The integration of Colibri with AxioVision, results in extremely fast switching times, with precise control of the illumination intensity to protect the sample.

For applications requiring higher illumination intensity, or for applications requiring excitation wavelengths not currently supported by existing LED technology, Carl Zeiss MicroImaging offers a combination system that pairs a Colibri with an externally coupled Metal Halide (HXP) white light source. The Colibri control panel or the AxioVision software is used to switch over to and control the shutter of the HXP 120.

\section{Summary}

White light sources have been in use for decades, and much expertise has been developed in the ways of reducing the problems associated with peripheral light. Many labs have invested a great deal in filters used to suppress non-specific light. In the past, it may have been difficult to imagine that any other light source method would ever be viable. Fortunately, a new type of light source is available. With LED technology, users can now take advantage of an excellent alternative for live cell imaging, high-speed or multi-channel fluorescence microscopy, and many other applications.

While the intensity of the LEDs has evolved significantly over the past few years, their intensity is still not as high as conventional arc lamps. However, in live cell imaging environments, intensity from conventional light sources are typically reduced to minimize phototoxic affects to cells and tissues.

\section{When people discover how well the Evactron ${ }^{\circledR}$ De-Comtaminator cleans SEMs they ask:}

Could You Make it Clean My Kitchen?

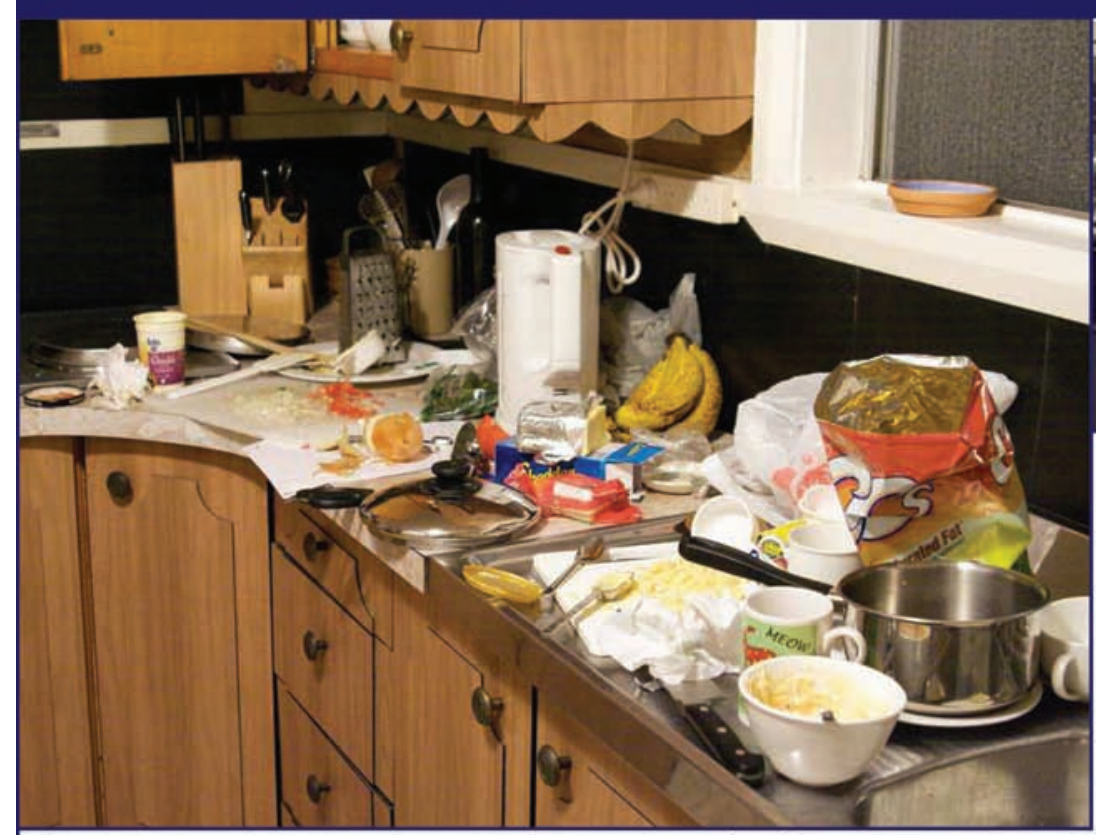

Electron Microscope operators are so impressed with Evactron D-C's gentle and effective cleaning power, they ask us if we can make a bigger Kitchen Evactron with the same powerful oxygen radicals that sweep out grease and oil from SEMs. Fantastic idea. We're considering it!

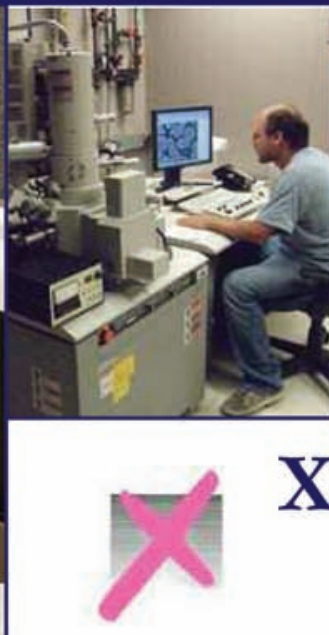

OVER 700

EVACtRons D-Cs SOLD WORLDWIDE TO CLEAN SEMs, FIBs, \& HIMs
New Oxygen Radical Source ORS: Now twice the cleaning power!

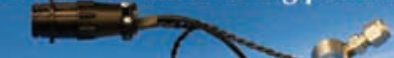

\section{.}

